\title{
Brain Radionecrosis: Case Report
}

\author{
Leandro Pelegrini de Almeida ${ }^{a}$, , Tobias Ludwig do Nascimento ${ }^{a}$, Luiz Pedro Willimann Rogerio ${ }^{a}$, \\ Marcelo Martins Reis ${ }^{\mathrm{a}}$, Guilherme Finger ${ }^{\mathrm{a}}$, Gabriel Frizon Greggianin ${ }^{\mathrm{a}}$, Fernanda De Carlia, \\ Gregori Manfroi ${ }^{\text {a }}$ Eduardo Anzolin ${ }^{\text {a }}$, Pasquale Gallo ${ }^{\mathrm{a}}$
}

\begin{abstract}
Brain necrosis is a possible complication caused by radiation therapy used in the treatment of head and neck cancer. This complication has variable neurological symptoms according to the site of brain damage, including motor deficits, aphasia, altered consciousness, seizures, and intracranial hypertension and also a variable time for presentation ranging from 3 months to 10 years. Once established, brain injury is irreversible and it is characterized by non-specific radiological image that submits the questions about its etiology: is it secondary to radionecrosis? Tumor progression? Primary tumor? The clinical report here described demonstrates the possibility of such differential diagnosis in a patient with brain lesion as a consequence of radiotherapy for a skin cancer.
\end{abstract}

Keywords: Radiotherapy; Necrosis; Brain diseases; Brain neoplasms; Squamous cell carcinoma

\section{Introduction}

The first report on cerebral necrosis induced by radiation was reported by Fisher and Holfelder in 1930 in a 45-year-old patient, treated with irradiation (68.4 Gy) for a temporal region basal cell carcinoma [1]. Most patients develop this degeneration after being treated with radiation therapy for brain or head and neck tumors [2].

This clinical report aimed to describe a case of cerebral radionecrosis after a radiation therapy for skin cancer, with a literature revision for discussing the differential diagnosis with primary or secondary brain cancers.

\section{Case Report}

A 69-year-old white Brazilian man looked for medical atten-

\footnotetext{
Manuscript accepted for publication October 17, 2016

aDepartment of Neurosurgery, Cristo Redentor Hospital, Porto Alegre, Rio Grande do Sul, Brazil

${ }^{b}$ Corresponding Author: Leandro Pelegrini de Almeida, Department of Neurosurgery, Cristo Redentor Hospital, Porto Alegre, Rio Grande do Sul, Brazil. Email: leandropelegrini@yahoo.com.br
}

doi: https://doi.org/10.14740/jnr396w tion for an erythematous and irregular macula on a left preauricular region on September 2012. He was submitted to a surgical resection on February 2013, with compromised deep margin, with the pathology being epidermoid carcinoma. Patient received complimentary treatment with radiation therapy in between April and June 2013 with 66 Gy (33 sessions of 2 Gy). In mid 2014, he started with a confusional state and motor aphasia. The enhanced magnetic resonance imaging (MRI) showed an irregular area of hypersignal with hypointense content on T1WI on insular lobe (Fig. 1a, b). He was submitted to a stereotactic biopsy in December 2014, which showed necrosis of cerebral tissue. He received corticotherapy and improved, but had another sudden worsening of same symptoms in July 2015. Emergency head CT showed worsening of cerebral edema in left temporal fossa with sings of intracranial hypertension (Fig. 2a, b). The worsening of symptoms were deviation of midline and an organized lesion that was approached for resection. After 2 days, he was in a coma, with anisocoria and worsening of hypodensity on head CT (Fig. 3a). A decompressive craniectomy was performed (Fig. 3b) and the pathology showed again just cerebral necrosis (Fig. 4). The outcome of patient showed a Glasgow outcome scale of 2 and the postoperative enhanced MRI showed the same findings of hyperintensity with hypodense content (Fig. 5).

\section{Discussion}

Late cerebral radiation necrosis can be a consequence of total brain radiation therapy that appears with doses up to $50 \mathrm{~Gy}$, but with higher incidences depending on doses, fraction and addiction of chemotherapy $[2,3]$. In a general way, its incidence varies from $0.5 \%$ to $25 \%$, with the peak within the third year from radiation therapy [4].

The findings are maculopathy and/or necrosis [4], but some of these are not due to radiation itself, but due to the mass effect of these lesions over the brain [5]. Among symptoms we can see cognitive deficits, hemiparesis, aphasia, seizures and any another depending on the cerebral topography, with tumor-like symptoms $[6,7]$.

It is well known that radiation therapy is more toxic for cells with higher mitotic levels. Saying that, on brain, the vascular endothelium and the oligodendrocytes can be more affected by radiation [4]. Vascular damage due to changes on endothelium induced by radiation leads to microvasculopathy, resulting in vascular insufficiency and infarction, followed by brain necrosis $[2,3]$. Late cerebral necrosis (months to years 

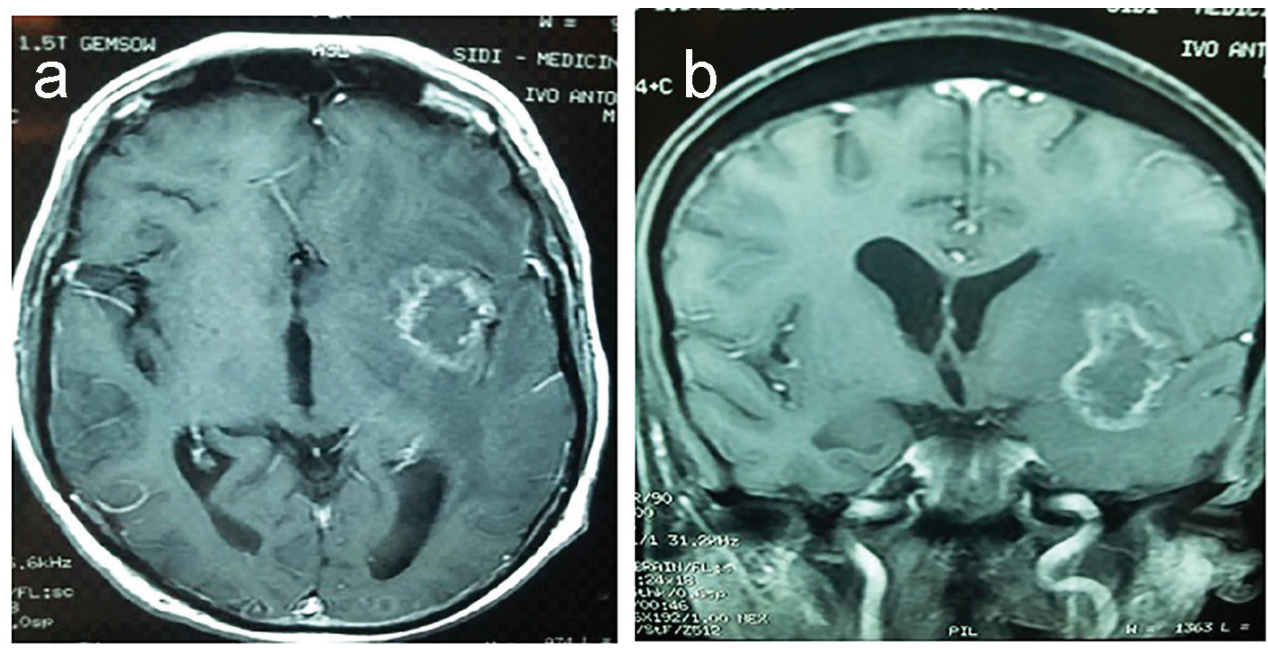

Figure 1. Enhanced T1WI MRI with a left insular lesion: (a) axial view; (b) coronal view.
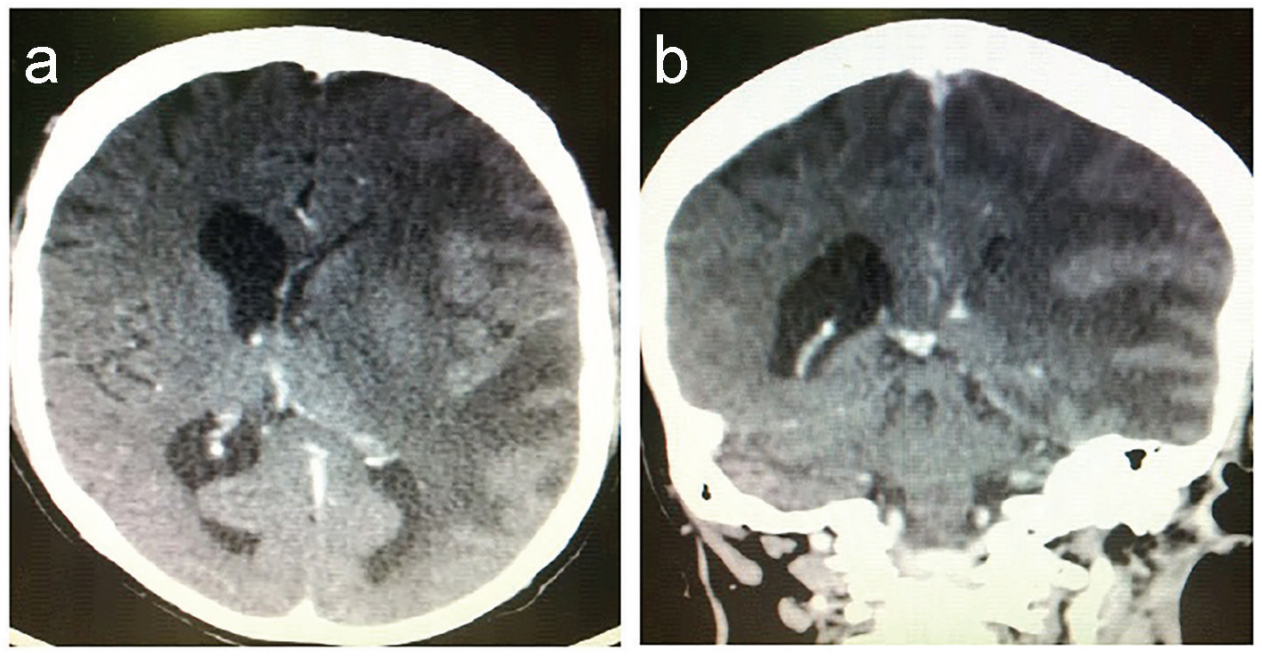

Figure 2. NECT of head with edema and midline shift: (a) axial view; (b) coronal view.
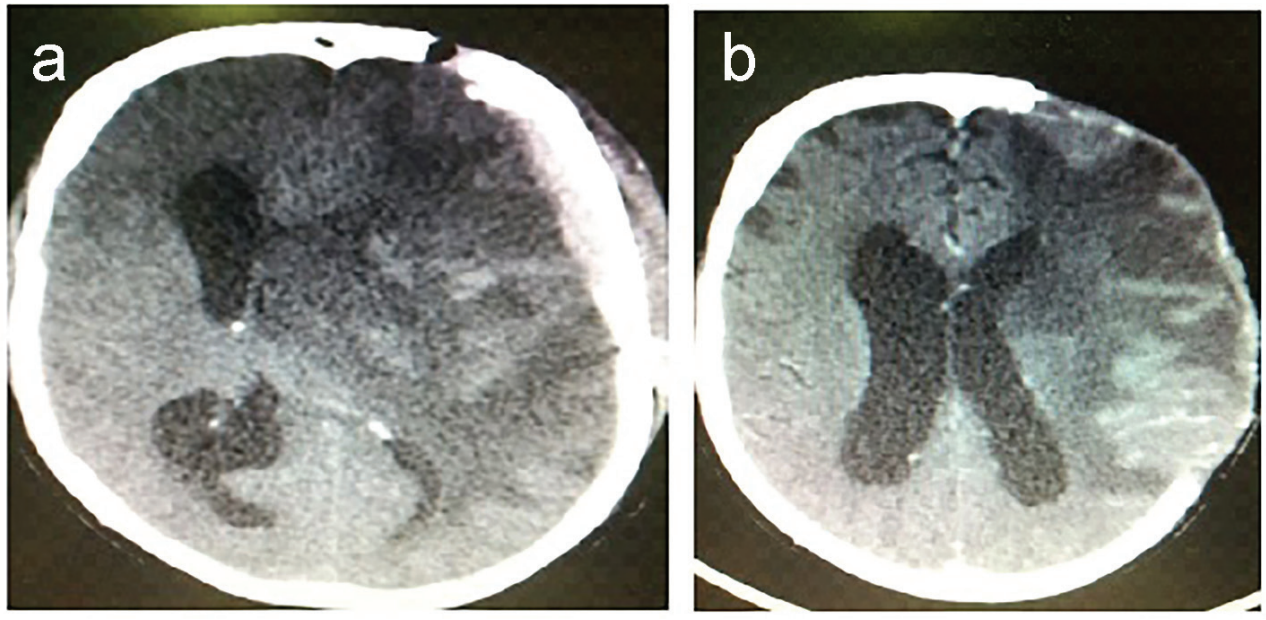

Figure 3. Postoperative NE head CT with (a) worse cerebral edema (axial view) and (b) decompressive craniectomy (axial view). 

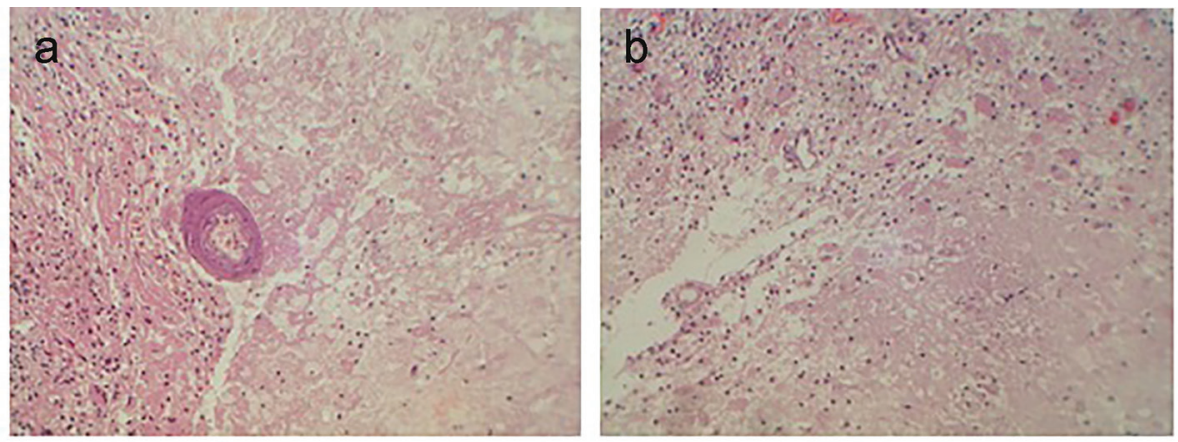

Figure 4. (a, b) Brain tissue with focus of necrosis (H\&E preparation).

after radiation therapy) is characterized by vasculitis [4], with pathological findings of sclerosis of microvasculature along with perivascular fibrosis and areas of petechial hemorrhage $[2-4,6,8]$.

Methods of brain imaging like head CT, MRI and PET-CT, although useful for cerebral radionecrosis, are not specific [1]. When associated with history of radiation therapy, interpretation becomes more reliable [8]. Some patterns like "Swiss cheese" and "soap bubble" are characteristics of cerebral radionecrosis on enhanced T1WI MRI [1,2]. Besides these well-known patterns, it can be doubtful when the site irradiated had previously a tumor [8]. The gold standard for that is histopathologic analysis $[1,2,4]$.

The treatment goals on radionecrosis, being a non-neoplastic condition, must be minimizing loss of neurological function, preventing progression and, if possible, reverting the pathological process [5]. Traditional treatment includes corticosteroids, antiplatelets, anticoagulants, high doses of vita-

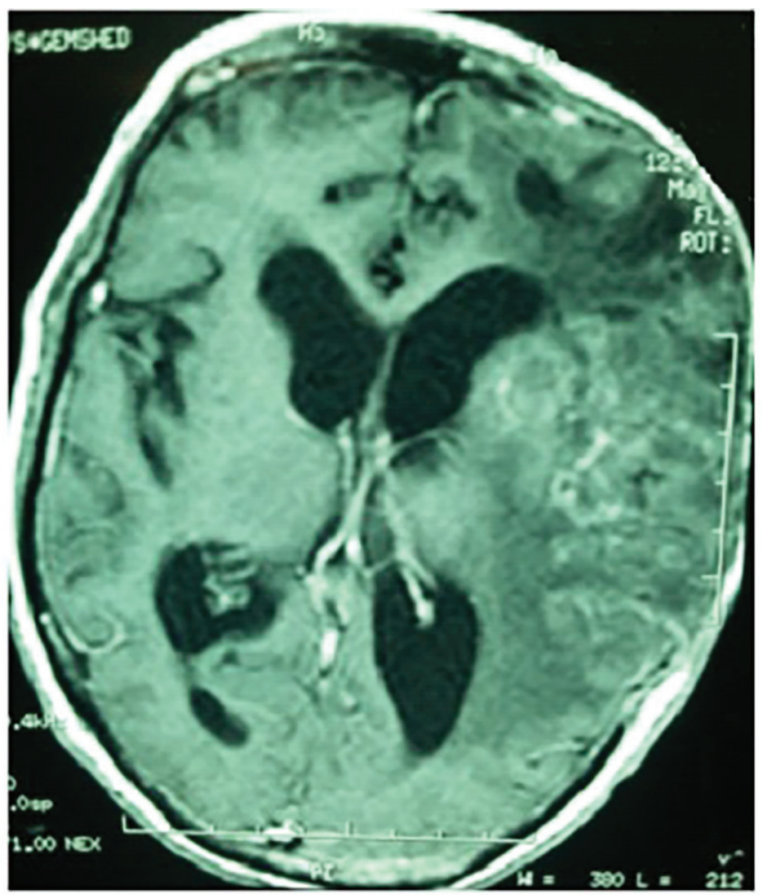

Figure 5. Postoperative enhanced T1WI MRI (axial view). mins and surgery $[1,2,5,6,9,10]$.

Surgery was always the last option on radionecrosis treatment, indicated only in large lesions, hemorrhagic complications or brain abscess $[5,8,9]$. Necrotic tissue resection along with decompressive craniectomy seems to improve prognosis of these patients, especially for those in emergency situations [10].

There is evidence today that justifies using bevacizumab, a humanized monoclonal antibody that inhibits vascular endothelial growth factor (VEGF), but still needs more investigational study to be a part of standard protocols [9, 11-13].

\section{Conclusion}

Cerebral radionecrosis carries morbidity and mortality as a complication from brain and head and neck tumors. Literature shows limited evidence of case reports or small series of cases about the efficiency of surgery on management of cerebral radionecrosis. It shows that surgical approach can revert only partially the process, with the goal of reducing intracranial hypertension by removing necrotic tissue and decompressing healthy brain.

\section{Conflicts of Interest}

The authors do not declare any conflicts of interest.

\section{References}

1. Dassarath M, Yin Z, Chen J, Liu H, Yang K, Wu G. Temporal lobe necrosis: a dwindling entity in a patient with nasopharyngeal cancer after radiation therapy. Head Neck Oncol. 2011;3:8.

2. Miyatake S, Nonoguchi N, Furuse M, Yoritsune E, Miyata T, Kawabata S, Kuroiwa T. Pathophysiology, Diagnosis, and Treatment of Radiation Necrosis in the Brain. Neurol Med Chir (Tokyo). 2015;55(Suppl 1):50-59.

3. Yoshii Y. Pathological review of late cerebral radionecrosis. Brain Tumor Pathol. 2008;25(2):51-58.

4. Lee JK, Chelvarajah R, King A, David KM. Rare presentations of delayed radiation injury: a lobar hematoma and 
a cystic space-occupying lesion appearing more than 15 years after cranial radiotherapy: report of two cases. Neurosurgery. 2004;54(4):1010-1013; discussion 1013-1014.

5. Wong ST, Loo KT, Yam KY, Hung WM, Fok KF, Yuen SC, Fong D. Results of excision of cerebral radionecrosis: experience in patients treated with radiation therapy for nasopharyngeal carcinoma. J Neurosurg. 2010;113(2):293300.

6. Giglio P, Gilbert MR. Cerebral radiation necrosis. Neurologist. 2003;9(4):180-188.

7. Vallee B, Malhaire JP, Person H, Colin J. Delayed cerebral pseudo-tumoral radionecrosis following scalp-tumour irradiation. Case report and review of literature. J Neurol. 1984;231(3):135-140.

8. Valk PE, Dillon WP. Radiation injury of the brain. AJNR Am J Neuroradiol. 1991;12(1):45-62.

9. Chen J, Dassarath M, Yin Z, Liu H, Yang K, Wu G. Radiation induced temporal lobe necrosis in patients with nasopharyngeal carcinoma: a review of new avenues in its management. Radiat Oncol. 2011;6:128.

10. Li Y, Shi X, Rong X, Peng Y, Tang Y. Neurosurgery and prognosis in patients with radiation-induced brain injury after nasopharyngeal carcinoma radiotherapy: a followup study. Radiat Oncol. 2013;8:88.

11. Gonzalez J, Kumar AJ, Conrad CA, Levin VA. Effect of bevacizumab on radiation necrosis of the brain. Int $\mathrm{J}$ Radiat Oncol Biol Phys. 2007;67(2):323-326.

12. Levin VA, Bidaut L, Hou P, Kumar AJ, Wefel JS, Bekele $\mathrm{BN}$, Grewal J, et al. Randomized double-blind placebocontrolled trial of bevacizumab therapy for radiation necrosis of the central nervous system. Int $\mathrm{J}$ Radiat Oncol Biol Phys. 2011;79(5):1487-1495.

13. Torcuator R, Zuniga R, Mohan YS, Rock J, Doyle T, Anderson J, Gutierrez J, et al. Initial experience with bevacizumab treatment for biopsy confirmed cerebral radiation necrosis. J Neurooncol. 2009;94(1):63-68. 